\title{
PERSPECTIVES OF THE EXPLICIT RETRIEVAL OF THE COMPLEX REFRACTIVE INDEX OF AEROSOLS FROM OPTICAL DATA TAKEN WITH LIDAR
}

\author{
Alexei Kolgotin ${ }^{1}$, Detlef Müller ${ }^{2,3}$, Eduard Chemyakin ${ }^{2}$, Anton Romanov ${ }^{4}$ \\ ${ }^{(1)}$ Physics Instrumentation Center, Troitsk, Moscow Region, 142190, Russia, alexeift@yahoo.com \\ ${ }^{(2)}$ Science Systems and Applications, Inc. (SSAI), NASA LaRC, Hampton, VA, 23666, USA, and \\ ${ }^{(3)}$ University of Hertfordshire, Hatfield, Hertfordshire, AL10 9AB, UK \\ ${ }^{(4)}$ The National University of Science and Technology, Leninskii av. 4, Moscow, 119049
}

\begin{abstract}
We developed an explicit approach for the retrieval of the complex refractive index from optical data, i.e. backscatter and extinction coefficients measured with lidar. In this approach we assume that we know the particle size distributions as well as the optical data. On the basis of this approach we carried out numerical simulations in order to test the uncertainty of the retrieval of the complex refractive index in dependence of the combination of extinction and backscatter coefficients, the measurement wavelengths and measurement errors.
\end{abstract}

\section{INTRODUCTION}

A number of methods for the retrieval of particle microphysical parameters from multiwavelength lidar measurements was developed in the past years [1-5]. The methods require us to apply a grid of complex refractive indices (CRI) as input; for example, real parts in this grid may range from 1.3 to 1.8 and the imaginary part may range from 0 to 0.05 . The final solutions of the CRI grid then are defined implicitly in the sense that the solutions fulfill a specific set of constraints and mathematical rules. For example, the constraint is applied that the optical data that follow from these solutions agree to the input optical data that were used in the retrieval. Besides that, the rule of averaging the solutions in the vicinity of the modified discrepancy minimum can be exploited as well [3]. In this contribution we explored an approach which allows us to derive the CRI explicitly from optical data (OD) taken with lidar. The particle size distribution (PSD) in our approach is assumed to be known.

In section 2 we present the methodology. In section 3 we carry out numerical simulations with synthetic optical data. We assume that the PSDs can be described by the log-normal law. Section 4 summarizes our results.

\section{METHODOLOGY}

OD such as extinction $\alpha(\lambda)$ and backscatter $\beta(\lambda)$ coefficients measured with lidar at wavelength $\lambda$ are related to the PSD $f(r)$ via integral equations of the form

$$
\int_{0}^{\infty} K_{g}(\lambda, m, r) f(r) d r=g(\lambda), \quad g=\alpha, \beta
$$

where the kernels of the integral equation $K_{g}(\lambda, m, r)$ are described by Mie theory in case of spherical particles of radius $r$ and CRI $m=m_{R}-i m_{I}$. In our previous work we investigated Eq. (1) to estimate the PSD $f(r)$ on the basis of input data $\alpha(\lambda)$ and $\beta(\lambda)$. If we assume that there is some estimation of $f(r)$ [2-3] then we can rewrite eq. (1) and use this estimation of $f(r)$ for the retrieval of $m$, i.e. we can write

$$
g(m)=g \quad \text { where } \quad g=\alpha, \beta
$$

and for fixed wavelength $\lambda$ we obtain

$$
g(m)=\int_{0}^{\infty} K_{g}(\lambda, m, r) f(r) d r, \quad g=\alpha, \beta
$$

This equation is a typical nonlinear algebraic equation and we define it on the domain

$$
m_{R} \in[1.3,1.8], m_{I} \in[0,0.099]
$$

Since we deal with the unknown parameter $m$ that depends on pairs of values $\left(m_{R}, m_{I}\right)$ we need to consider Eq. (2) two times, i.e. for the different data types $\alpha$ and $\beta$ or at the different wavelengths $\lambda_{1}$ and $\lambda_{2}$ in order to make the problem determinate. As a result we obtain two unknown parameters $m_{R}$ and $m_{I}$ for two nonlinear algebraic equations which theoretically can be solved.

Unfortunately, an analytical solution of the system (2) is not possible. For that reason we reformulate this problem in another way. Let us compute three different kinds of discrepancies:

$$
\begin{array}{cr}
\rho_{g}^{2}(\mathbf{m})=[g-g(\mathbf{m})]^{2} / g^{2} & g=\alpha, \beta(5) \\
\rho_{t}^{2}(\mathbf{m})=\left[\rho_{\alpha}^{2}(\mathbf{m})+\rho_{\beta}^{2}(\mathbf{m})\right] / 2 & (6)
\end{array}
$$

The vector $\mathbf{m}$ denotes the pair $\left(m_{R}, m_{I}\right)$. In that case, the solution of the system (2) can be treated as a minimization problem for the discrepancies 


$$
\rho_{j}^{2}(\mathbf{m})=\rho_{j}^{2}\left(m_{R}, m_{I}\right) \rightarrow \min \quad j=\alpha, \beta, t
$$

where we consider the constraint used in (4).

There are plenty of approaches to minimize the functions $\rho_{j}(\mathbf{m})$ : gradient method, random search, method of cyclic descent (MCD) and so on [6]. In view of the information we have about the kernel functions we use the MCD in our investigation.

This method of function optimization does not require us to calculate the derivative and one of the modifications of the MCD used here can be described by the following algorithm:

1 . We define an arbitrary initial value, for example, $\mathbf{m}^{0}=(1.3 ; 0)$

2. One of the two unknown parameters is fixed, for example, $m_{R}{ }^{0}$ and we consider the onedimensional minimization problem for $\xi$ :

$$
\rho_{j}^{2}\left(m_{R}^{0}, \xi\right) \rightarrow \min \quad j=\alpha, \beta, t
$$

on the domain $\xi \in[0,0.1]$. This problem can be solved, for example, with the golden section method [6]. After this step the $1^{\text {st }}$ approximation for the parameter $m_{I}$ is found as

$$
m_{I}^{1}=\xi^{*},
$$

where $\xi^{*}$ is the solution of problem (8).

3. Another parameter is fixed, in our case $m_{I}{ }^{1}$, and the one-dimensional minimization problem is considered again for $\xi$,

$$
\rho_{j}^{2}\left(\xi, m_{I}^{1}\right) \rightarrow \min \quad j=\alpha, \beta, t(10)
$$

on the domain $\xi \in[1.3,1.8]$. After this step the $1^{\text {st }}$ approximation for the parameter $m_{R}$ is found, i.e.,

$$
m_{R}{ }^{1}=\xi^{*},
$$

where $\xi^{*}$ is the solution of problem (10).

4. We compare the two points $\rho_{j}\left(m_{R}^{0}, m_{I}^{0}\right)$ and $\rho_{j}\left(m_{R}{ }^{1}, m_{I}{ }^{1}\right)$. If the deviation $\Delta \rho_{j}$

$\Delta \rho_{j}=\left|\rho_{j}\left(m_{R}{ }^{1}, m_{I}{ }^{1}\right)-\rho_{j}\left(m_{R}{ }^{0}, m_{I}{ }^{0}\right)\right|<\varepsilon j=\alpha, \beta, t(12)$

is fulfilled at the preliminarily initialized threshold $\varepsilon$ the iteration process is stopped. Otherwise steps 1-4 should be repeated.

5 . With the last iteration $n$ we obtain the solution $\mathbf{m}^{*}=\mathbf{m}^{n}$ from problem (7).

The minimization procedure including the steps 1-5 formalizes our explicit approach to the retrieval of the CRI from a set of OD measured with lidar.

\section{NUMERICAL SIMULATION}

We used our approach of retrieving the CRI with synthetic OD that were computed directly with Eq. (1) for known CRI and PSD $f(r)$ which is described by the lognormal law. We used a range of values for the mean radius $r_{0} \in[0.1,0.3] \mu \mathrm{m}$ and the mean width $\sigma \in[1.3 ; 2.0]$ (defined accord- ing to the lognormal law) and we computed synthetic OD for different $\lambda$. Here we present some results of the CRI retrievals on the basis of a lognormal PSD with $r_{0}=0.22 \mu \mathrm{m}, \sigma=1.65$ and $m=1.4-i 0.0019$. It is not a very special case. However it illustrates quite well the major particularities of the problem of CRI retrievals.

First, we tested OD sets that consist of 1-3 backscatter coefficients (corresponding to measurement wavelengths of 355, 532, and $1064 \mathrm{~nm}$ ) and 1-2 extinction coefficients (corresponding to measurement wavelengths of 355 and $532 \mathrm{~nm}$ ). We show a few examples of our studies.

As we discussed in section 2 we need at least two coefficients to make our problem determinate. We considered in our first simulation the input OD that contain only backscatter $(2 \beta)$ or only extinction $(2 \alpha)$ coefficients at $\lambda_{1}=355 \mathrm{~nm}$ and $\lambda_{2}=532 \mathrm{~nm}$. Unfortunately, the minimization procedure for the functionals $\rho_{\beta}(\mathbf{m})$ and $\rho_{\alpha}(\mathbf{m})$ failed for these combinations of input OD. This result agrees well with previous studies [2, 3]. If we include the backscatter coefficient at $\lambda_{3}=1064 \mathrm{~nm}$ we do not find a significant improvement of the results based on this set of $3 \beta$. The retrieval results for the input set $1 \beta+1 \alpha$ $(\lambda=532 \mathrm{~nm})$ are shown in Fig. 1: the discrepancies $\rho_{t}(\mathrm{a}), \rho_{\beta}(\mathrm{b})$ and $\rho_{\alpha}(\mathrm{c})$ are shown in dependence of the parameters $m_{R}$ and $m_{l}$. The minimum values close to $0 \%$ correspond to the true CRI (red circle) on these maps. The maximum values are $22.6 \%$ for $\rho_{\alpha}$ and $1180 \%$ for $\rho_{\beta}$. A very important and typical property of the two surfaces shown in Fig. $1 \mathrm{~b}$ and $1 \mathrm{c}$ is that they contain the light areas ("anti-gradient" direction) with smallest values $(0 \%-1 \%)$ distributed from the point $m=1.4-i 0.0019$ to the point $m=1.8$ $i 0.099$ for $\rho_{\beta}$ and from the point $m=1.4-i 0.0019$ to the point $m=1.55-i 0.099$ for $\rho_{\alpha \text {. }}$. Apparently it results from the properties of the kernel functions $K_{\beta}(\lambda, m, r)$ and $K_{\alpha}(\lambda, m, r)$.

To extract in a better way the behavior of the three surfaces near the minimum values we connected the 5 points with solid lines along the smallest discrepancies. Unfortunately, the lines are spread out along all anti-gradient directions. It means that even a small averaging interval of the discrepancy [3] can lead to the large variations of the CRI in the range (4).

Fig. 1 shows the CRI (big blue close circle) retrieved with our minimization procedure described in section 2 . In that procedure we used the initial values $\mathbf{m}^{0}=(1.8 ; 0.0)$ and $\varepsilon=10^{-13}$. After 
$n=22$ iterations we obtained the solution $\mathbf{m}^{*}=\mathbf{m}^{22}=(1.4 ; 0.001898)$. If we ask for more accuracy it can be improved. For example, for $\varepsilon=10^{-14}$ we obtain $\mathbf{m}^{*}=\mathbf{m}^{28}=(1.4 ; 0.001899)$. The convergence speed depends on the behavior of the discrepancy surface: the more pronounced the optimum is the faster the convergence is. It is also defined by the PSD and the wavelength.

The next important question concerns the conditionality of our problem (7); in other words, what is the stability of this solution scheme with regard to different errors? Here we consider this question just with respect to the OD measurement error $\delta g$.

We could employ our minimization procedure for different realizations of that error for a fixed OD set in order to investigate the uncertainty of the CRI retrieval $\delta m$. However it can be seen immediately from the maps for $\rho_{t}, \rho_{\beta}$ and $\rho_{\alpha}$. For that reason we plotted in Fig. 1 the small open circles which show the area where the discrepancy $\rho_{j} \leq 10 \%$ reflects a measurement error of $10 \%$.

As we can see solutions corresponding to that area are spread along all anti-gradient directions from $m=1.4-i 0.0019$ to $m=1.8-i 0.099$. The results do not improve if we take into account other combinations of OD that can be measured by lidar, for example, $3 \beta+2 \alpha$. Recent studies [7] also demonstrate that extra constraints are necessary for accurate estimations of the CRI.

We therefore investigated options that would allow us to improve the CRI retrieval. We considered measurement scenarios in which we might have another extinction value at a wavelength above $532 \mathrm{~nm}$. We are not aware of lidars that measure extinction at $1064 \mathrm{~nm}$. However, we could think of a measurement configuration in which we combine lidar with a passive remote sensor that measures at around $1064 \mathrm{~nm}$ or a longer wavelength. For example AERONET sunphotometer provides optical depth at 1640 $\mathrm{nm}$. We could use lidar profiles to derive profiles of extinction from optical depth measured at this longer wavelength. Previous feasibility studies have shown how this could be done, e.g., Balis et al. [9]. We show the results for the scenario in which we have extinction at $1064 \mathrm{~nm}$.

Fig. 2 shows results for $1 \beta+1 \alpha$ at $\lambda=1064 \mathrm{~nm}$. The extinction anti-gradient changes the direction significantly, and for $10 \%$ measurement error the CRI retrieval is still accurate to $\pm i 0.013$ for the imaginary part and to- \pm 0.025 for the real part (see small open circles in Fig. 2a). If we use extinction at $2 \mu \mathrm{m}$ we obtain the imaginary part to an accuracy of $i \pm i 0.004$ and the real part to an accuracy of \pm 0 .

We note one more result of our analysis. A recent publication [8] demonstrated a specially developed approach to extracting the real part of the CRI. Our study shows that if we can estimate either the real or the imaginary part of the CRI we can derive immediately the other part of the CRI on the basis of the anti-gradient direction area that is shown in the $\rho_{t}$ map. For example, if in our simulation case we would constrain the real part within the range $[1.40 ; 1.45]$ the imaginary part range could be constrained to the interval [0;0.015] (see Fig. 1a).

\section{CONCLUSION AND SUMMARY}

We present an approach for the retrieval of the CRI in an explicit way from OD taken with lidar. We carried out numerical simulations in order to test the capabilities of this method.

Our approach allows us to estimate the CRI with any pre-assigned accuracy if the OD and the PSD are known. However the method can be employed only for the case of combinations of backscatter and extinction coefficients.

We investigated the uncertainty of the CRI retrievals in dependence of the measurement error. We showed that we can achieve an accuracy of $\pm i 0.05$ for the imaginary part and \pm 0.3 for the real part if the uncertainty of the OD is $10 \%$ and if we do not apply any other constraints to the investigated parameters.

The accuracy of the CRI retrieval can be improved significantly if, for example, extinction coefficients from optical depth measurements at longer wavelengths are used. Data at longer wavelengths could for instance be provided by a combination of lidar with passive remote sensing. The example for $1064 \mathrm{~nm}$ shows that the uncertainties of the real and imaginary parts decrease to \pm 0.025 and $\pm i 0.013$, respectively, even for measurement errors of $10 \%$.

We will develop our approach further in order to investigate the influence of incorrectly given PSD on the uncertainty of the CRI retrieval. 

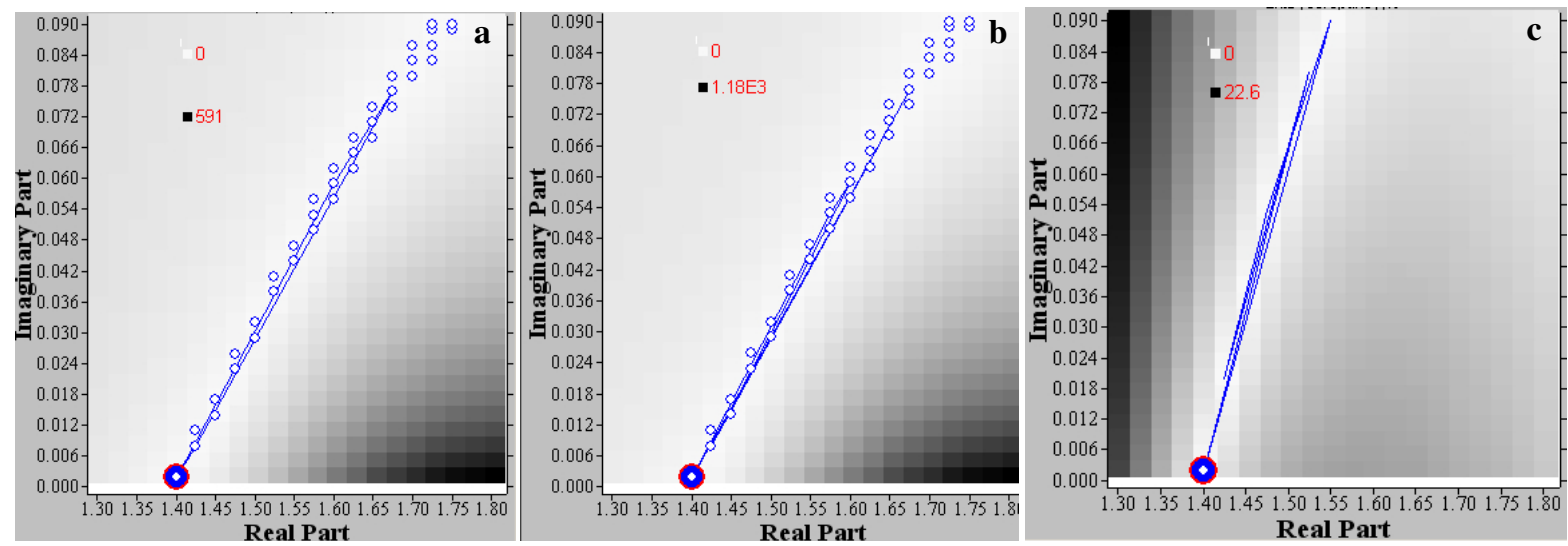

Fig. 1. Discrepancies $\rho_{t}(\mathrm{a}), \rho_{\beta}$ (b) and $\rho_{\alpha}$ (c) versus $\left(m_{R}, m_{I}\right)$ for the OD set $1 \beta+1 \alpha$ at $\lambda=532 \mathrm{~nm}$. Big close red and blue circles show the true values and the retrieved values of the CRI, respectively. The solid line connects the five points that are obtained from the smallest discrepancy values which are less than $1 \%$. The small open circles are the retrieved CRIs if the OD error varies from 0 to $10 \%$ (not shown for $\rho_{\alpha}$ ). Legend's black square means $591 \%$ (a), $1180 \%$ (b) and $22.8 \%$ (c)
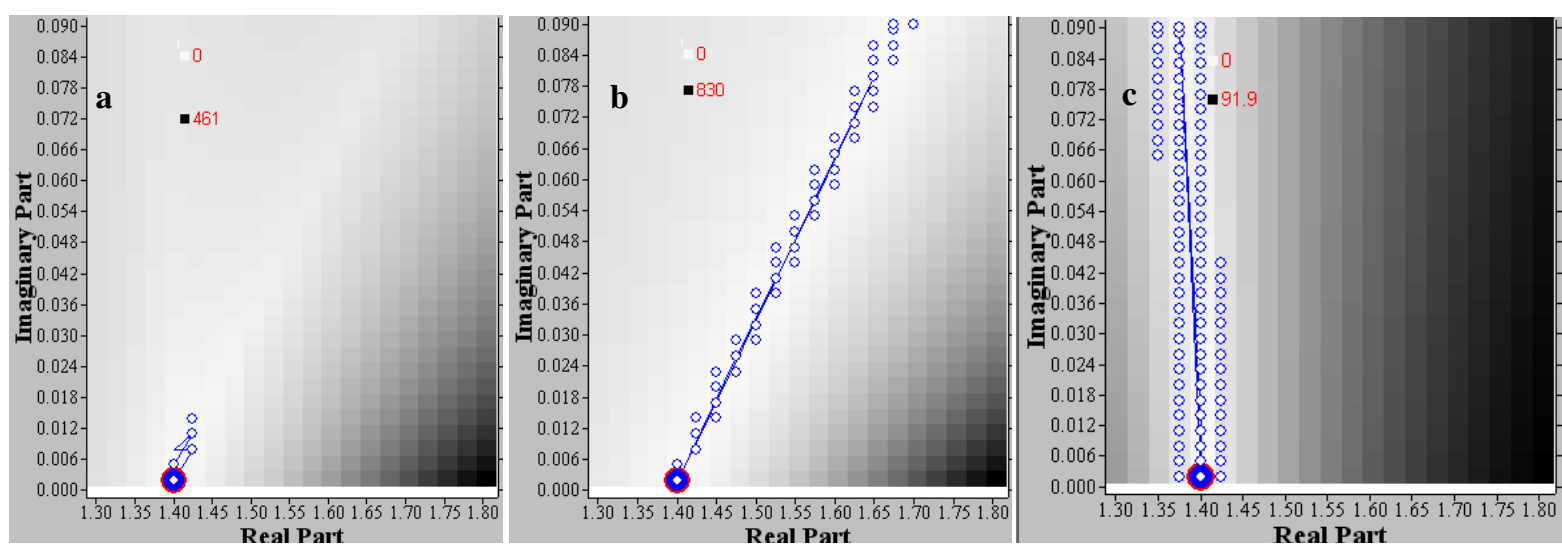

Fig. 2. Same as Fig. 1. but the OD set $1 \beta+1 \alpha$ includes the backscatter and extinction coefficients at $\lambda=1064 \mathrm{~nm}$.

\section{REFERENCES}

1. Donovan D., A. Carswell, 1997: Principal component analysis applied to multiwavelength lidar aerosol backscatter and extinction measurements, Appl.Opt.36, pp. 9406-9424.

2. Müller D., et al., 1999: Microphysical particle parameters from extinction and backscatter lidar data by inversion with regularization: theory, Appl. Opt., 38, pp. 2346 - 2357.

3. Veselovskii I., et al., 2002: Inversion with regularization for the retrieval of tropospheric aerosol parameters from multiwavelength lidar sounding, Appl. Opt., 41, pp. 3685 - 3699.

4. Böckmann C., et al, 2005: Microphysical aerosol parameters from multiwavelength lidar, Journal of Optical Society of America, A, 22, pp. 518-528.

5. Chemyakin E., et al, 2014: "Arrange \& average" algorithm for the retrieval of aerosols parameters from multiwavelength HSRL/Raman lidar data, Appl. Opt., 53, pp. $7252-7266$.

6. P. E. Gill, W. Murray and M. H. Wright, 1982: Practical Optimization. Emerald Publishing).
7. . Veselovskii I., et al, 2005: Information Content of Multiwavelength Lidar Data With Respect to Microphysical Particle Properties Derived From Eigenvalue Analysis, Appl. Opt. 44, 5292-5303.

8. Veselovskii I., et al, 2012: Linear estimation of particle bulk parameters from multiwavelength lidar measurements, Atm. Meas. Tech., 5, 1-30.

9. Balis, D., E. Giannakaki, D. Müller, V. Amiridis, K. Kelektsoglou, S. Rapsomanikis and A. Bais (2010). "Estimation of the microphysical aerosol properties over Thessaloniki, Greece, during the Scout-O3 campaign with the synergy of Raman lidar and sunphotometer data." Journal Geophysical Research 115: D08202, doi:10.1029/2009JD013088. 